\title{
Mediating Factors in the Arousal-Performance Relationship
}

\author{
John A. Bargh ${ }^{1,2}$ and Jerry L. Cohen \\ University of Illinois at Urbana-Champaign
}

The arousal-performance relationship was investigated within a social facilitation experiment, in which two major task dimensions (cognitivemotor and difficulty) were manipulated and two arousal measures (palmar sweat and self-reports) were taken. Subjects (75 male and 75 female introductory psychology students) were randomly assigned to one of five audience conditions and one of three task difficulty levels. Each subject performed three tasks, which varied as to cognitive and motor requirements, under a uniform difficulty level. No significant differences were found on any arousal or performance measure due to the audience manipulation. Females were found to be more aroused by the audiences than males, on both arousal measures. When self-reported arousal scores were quintiled to create five post hoc arousal conditions, significant interactions between these conditions and task difficulty level were obtained for both the cognitive and motor tasks. (No significant differences were found using quintiled palmar sweat scores.) On the basis of the similar pattern of these interactions, it was concluded that the inverted- $U$ function was obtained only on high-difficulty tasks.

The effect of arousal on performance has long been a prime research topic. With Zajonc's (1965) theory of social facilitation, investigators of the effects of the presence of an audience on an individual's performance joined with those interested in physiological processes and related areas in active exploration of the arousal-performance relationship.

Zajonc (1965) concluded that the presence of an audience serves to increase the probability of the dominant response, i.e., social facilitation or inhibition. The form of this relationship between the level of arousal and

'John Bargh is now affiliated with the University of Michigan.

${ }^{2}$ Requests for reprints should be addressed to Mr. John A. Bargh, 3023 Institute for Social Research, Box 1248, Ann Arbor, Michigan 48106. 
task performance is assumed to be increasing monotonic; i.e., as drive increases, so does performance. This is the drive theory hypothesis.

Other theorists claim that the shape of the function between arousal and performance is an inverted $\mathrm{U}$ (the inverted-U hypothesis). That is, there exists a certain level of arousal which facilitates optimal performance, and greater or lesser levels result in poorer performance (Innes \& Young, 1975; Katahn, Blanton, \& Gipson, 1967; Martens \& Landers, 1972; Sorce \& Fouts, 1973; Stennett, 1957).

The concept of arousal is postulated to be an intermediate link between the effect of the presence of others and an individual's performance. The drive theory hypothesis states that the presence of the audience increases the individual's drive level, and that as the drive level increases, the probability of the emission of the dominant response increases. When the dominant response is the correct response, performance and drive level have a positive linear relationship. The inverted-U hypothesis asserts that increasing the level of arousal in the performing individual facilitates the quality of the performance up to an optimum, after which additional arousal causes a decrease in quality.

The inverted-U hypothesis does not rule out a positive linear relationship under certain circumstances, and can also accommodate the literature supporting an optimal arousal level; thus it supersedes the drive theory hypothesis. But since we cannot know the endpoints of the arousal continuum, the inverted-U hypothesis cannot be refuted (Martens, 1974). Evidence of a linear arousal-performance function can always be explained by claiming that the arousal level was not high enough and that higher levels are needed for a performance decrement. "Consequently, a more fruitful strategy at this point may be not to view the inverted- $U$ hypothesis as being correct or incorrect but instead to regard it as an issue of specifying the parameters for when it is correct"' (Martens, 1974, p. 178).

Social facilitation is conceptualized to be a three component process: audience characteristics, arousal, and performance. The main finding of research into audience characteristics is that the evaluative potential of an audience, as perceived by the subject, is the main contributor to increases in the subject's arousal level (Cottrell, 1968; Cottrell, Wack, Sekerak, \& Rittle, 1968; Henchy \& Glass, 1968; Paulus \& Murdoch, 1971; Sasfy \& Okun, 1974). Further, the higher this potential is perceived to be, the higher the level of arousal induced within the individual (Cohen \& Davis, 1973).

The exact relationship between the presence of an audience and arousal level is not known at this time, for no study has examined the effects of a constant audience condition on the different aspects of arousal. Until this is done we cannot directly compare experiments which measure different arousal dimensions. 
Other than Yerkes and Dodson's original 1908 work, there has been little research on the effects of task characteristics on the arousal-performance function given constant audience characteristics. Yerkes and Dodson (1908) found the optimal strength of the electrical stimulus for difficult tasks was lower than for simple tasks. This finding was replicated later by Broadhurst (1959) and McLaughlin and Eysenck (1967). Hackman (1969) argued in his analysis of the nature of tasks and their behavioral implications that task analysis is crucial to the complete understanding of experimental results:

Tasks to be used in behavioral research should no longer be considered merely "something for the subject to do" while other phenomena are being studied. For as long as this practice continues to be acceptable, important portions of the variability of subjects" reactions to experimental situations will continue to be ignored, with unfortunate consequences for both the interpretability and the generalizability of our results.... A high priority research need is the development of understanding about what "types" of task dimensions have substantial behavior impact, what the nature of this impact is, and how it interacts with various experimental treatments [p. 123].

\section{Plan of the Study}

Each of the three basic components of social facilitation effects (evaluative potential of audience, arousal measurement, and task characteristics) was manipulated. Five audience conditions were employed (ordered from hypothesized lowest to highest evaluative potential, based on Cohen \& Davis, 1973): no audience, observe-live audience, evaluative-live audience, videocamera-observe-live audience, and videocamera-evaluative-future audience. Both a physiological (the Palmar Sweat Index; Johnston \& Dabbs, 1967) and a self-inventory arousal measure (Spielberger, Gorsuch, \& Lushene, 1970) were employed.

Two characteristics of the task, difficulty and cognitive-motor requirements, were also manipulated. Subjects performed three tasks, differing as to their cognitive and motor requirements, at a uniform difficulty level (low, medium, or high). Sex of subject and trait anxiety level (high or low) were also included as attribute factors in the design.

The use of the five different audience compositions was thought to sample a broad enough region of the arousal continuum to test adequately the inverted-U hypothesis. The purpose of this study was thus to provide direct evidence of the validity and scope of the drive theory and inverted- $U$ hypotheses over a wide range of arousal and task dimensions, and also to investigate the possible mediating effects of arousal measurement and task characteristics on the arousal-performance relationship. 
By dichotomizing subjects into either high- or low-trait-anxiety categories, two earlier studies of trait anxiety and performance were conceptually replicated. Carron (1965) found an interaction between trait anxiety and task difficulty: high-trait-anxious subjects performed better than lowtrait-anxious subjects on the low-difficulty version of a motor task, and low-trait-anxious subjects performed better than high-trait-anxious subjects on the high-difficulty version. Scanlan (1975) reported that high-traitanxious subjects were generally more aroused by audiences than were the low-trait-anxious subjects.

It should be emphasized that this study is an attempt to determine the influence of several possibly important variables in social facilitation research in order to gain a greater understanding of the arousal-performance relationship. Beyond providing a more general test of the inverted- $U$ and drive theory hypotheses, this experiment does not test a particular theoretical position. Its purpose is exploratory in nature, and is intended to specify the influence of several important variables which have been to now largely ignored.

\section{METHOD}

\section{Subjects}

One hundred and fifty undergraduate students $(75$ males and 75 females) enrolled in the introductory psychology course at the University of Illinois participated in the experiment as partial fulfillment of a course requirement. Subjects ranged in age from 18 to 21 years, and participated in the experiment one at a time.

\section{Stimulus Materials}

Three tasks were presented to each subject: the Minnesota Pegboard test, the submarine game, and the WAIS blocks test.

Minnesota Pegboard. The pegboard (Green \& Berman, 1936) consisted of a wooden board, $20 \mathrm{~cm} \times 3 \mathrm{~cm} \times 53 \mathrm{~cm}$, containing 15 rows of 4 wooden pegs each (the original Minnesota Rate of Manipulation test employed blocks instead of pegs). Each cylindrical peg was approximately 5 $\mathrm{cm}$ long and $2.5 \mathrm{~cm}$ in diameter, and had one end painted black and the other left uncolored.

The pegs were arranged so that all had the same colored end visible (either black or uncolored). Subjects were instructed to move all of the pegs to an adjacent receptable board as quickly as they could using one hand 
only; their score would be the number of seconds it took to move all 60 pegs to the other board, the less time taken the better the score. For the warm-up trial, subjects used their preferred hand, and were told to move the pegs directly to the other board (without turning them over first) as quickly as possible.

The directions for the performance trial varied for the three difficulty conditions. The low difficulty version required moving the pegs with the nonpreferred hand directly to the receptacle board. For the medium difficulty condition, subjects used their preferred hand but had to turn the pegs over before placing them in the receptacle board. Subjects in the high-difficulty condition were instructed to use their nonpreferred hand and to turn the pegs over first. The validity of the ordering of these a priori difficulty conditions was confirmed by pretesting.

Submarine Game. The submarine game (Shaw, 1973, p. 117) employed different contour maps, one each of low, medium, and high difficulty as determined by pretesting in a pilot study. Each map consisted of a target coordinate and a contour system around that target. The object of the task was to locate the target in as few guesses as possible. A numerical value given by the experimenter after each guess served as feedback as to the accuracy of the guess. The higher this feedback was in value, the closer (in general) the guess was to the target coordinate. It was emphasized to the subjects that this value was only to help guide them in making their next guess, and had nothing to do with their score for the trial. The actual performance score was the number of guesses taken to locate the target. There was no time limit for guessing.

WAIS Blocks Test. This was the standard WAIS blocks test (Wechsler, 1955) in which subjects were required to manipulate the blocks to match a target pattern. Three different target patterns of low, medium, and high difficulty (as determined by pretesting) were employed. The score for a particular trial was the amount of time taken to complete the given pattern.

\section{Arousal Measures}

The physiological and self-inventory arousal measures were taken simuitaneously after the experimental instructions and between the warmup and performance trials of each task. The scoring procedure of the palmar sweat index (PSI), which gauged the amount of physiological arousal present, had an interjudge reliability of .905 .

The A-State scale of the Spielberger State-Trait Anxiety Inventory served as the self-report arousal measure. In addition, the A-Trait scale was administered to each subject prior to the main experimental session. 


\section{Experimental Design}

Ten subjects (five males and five females) were randomly assigned to each of the fifteen conditions formed by the basic $5 \times 3 \times 2$ design (audience composition, task difficulty, and sex of subject). In addition, repeated measures were taken on the cognitive vs. motor task requirements factor (each subject performing all three tasks at one difficulty level), and on the arousal measure factor (both measures were taken for every subject). Trait anxiety (high or low) was the final factor examined, although its relationship to the other factors could not be experimentally manipulated as scores could be dichotomized only after all subjects had participated. Thus, the experiment employed a $5 \times 3 \times 2 \times 3 \times 2 \times 2$ repeated measures factorial design.

\section{Procedure}

Subjects were first given the A-Trait scale to complete in a waiting room; when they had finished, they were shown into the experimental room, approximately 10 meters down the hall. In the no audience condition, curtains had been drawn covering a one-way mirror, and the subject was seated at the table facing the curtains. In the two live audience conditions the one-way mirror was exposed, and the subject was seated facing the mirror. Those in the observe-live condition were told that students in a psychology research course would be observing the experiment from behind the one-way mirror. The evaluate-live condition subjects were informed that students from another introductory psychology course would be evaluating their performance on several tasks from their vantage point behind the one-way mirror. These subjects were then shown a "rating form," consisting of several questions concerning the subject's skill, nervousness, and coordination, on which they were to be evaluated.

A videocamera mounted on a tripod, a television monitor, and a videotape recorder were set up in one corner of the room for the two videocamera audience conditions. In the videocamera-observe-live condition, the subject was told that students from a psychology research methods course were in the adjacent room and were going to watch the experiment on a television set identical to the monitor. An electrical cable could be seen running into the other room from the back of the monitor. The videotape was removed from the recorder while the experimenter told the subject that they were not going to record his/her performance, but allow the other room to monitor it.

In the videocamera-evaluate-future audience condition, the videotape recorder was started and subjects were told that their performance was 
being recorded in order for a group of students in another introductory psychology course to view the tape at a later date. These students were said to be serving as raters of the subject's performance, and subjects were shown the rating sheets described above.

There were no actual audiences present in the audience conditions; this necessitated the simulation devices described above. It should be noted that only one of the 150 subjects suspected the nonexistence of an audience during postsession discussion with the experimenter. Apart from practicality, the advantage of employing an unseen audience is that it has been shown to be more arousing than a visually present one (Wapner \& Alper, 1952).

In all conditions, the experimenter next explained that he was investigating human performance on three different types of tasks: one requiring mental operations, one which required only manual manipulation, and a third which combined elements of both. Subjects were told that they would be performing all three of the tasks, and that they would be asked periodically to complete a questionnaire concerning their reactions to the task they were involved in at the time.

The experimenter then explained the purpose of the PSI and demonstrated the procedure to the subject, applying the solution and letting it dry while the first A-State scale was administered (these were the pretask measures).

Subjects then performed the three tasks, all at the same randomly determined difficulty level. The presentation order of the tasks was randomized for each subject to control for any carryover effect from one task to the next.

After completion of the last task, the experimenter debriefed the subject and asked for his or her reaction to various aspects of the experiment. The subject was thanked for participating and was shown out of the experimental room.

\section{Dependent Variables}

Each subject had a performance score for each of the three tasks, two sets of arousal scores, and a score on the trait anxiety inventory. Performance on both the pegboard and the WAIS blocks tasks was measured by the number of seconds taken to complete the task. The submarine game task score was the number of guesses taken by the subject to find the target coordinate.

Both the PSI and A-State inventory were taken four times during the experimental session: after the experimental instructions and between the warm-up and performance trials of each task. Scores on the PSI, being 
direct counts of the active sweat glands visible in a subject's fingerprint, were not limited in range; the A-State scale ranged from 20 (lowest arousal) to 80 (highest arousal). In addition, each subject had a score on the A-Trait scale, which had the same range restrictions as the A-State scale.

\section{RESULTS}

The overall plan of the data analyses was to focus on the arousal-performance relationship, determining its general characteristics and any modifying influence of experimental variables. First, the arousal-producing capacity of each task was compared, followed by an examination of the effects of sex of subject and trait anxiety level on arousal and performance. The arousal-performance relationship was then assessed for each task through analyses of variance and trend analyses. From these tests the effect of task difficulty could be determined, and whether or not this effect differed over the cognitive-motor dimension.

\section{Comparison of Task Arousal Levels}

The arousing properties of each task relative to the other two were assessed with a repeated measures one-way analysis of variance, with task arousal measure as the factor (submarine game, blocks test, and pegboard). A significant main effect was found on both the PSI and A-State scores $[F(2,288)=7.10, p<.001$ for the PSI; $F(2,298)=16.07, p<.001$ for the A-State]. The mean arousal score for the blocks test is higher than that of the pegboard task, which elicited more arousal than the submarine game, for both arousal measurement techniques. As shown in Figure 1, the patterns of the two standardized sets of arousal scores (A-State and PSI) over the cognitive-motor dimension (ordered by increasing motor and decreasing cognitive requirements) are very similar.

\section{Sex Differences}

To test for sex differences in arousal over the experiment in general, the A-State and PSI scores were each averaged over the three task arousal measurements and then submitted to separate $3 \times 2$ analyses of variance, with difficulty level and sex of subject as factors. For both the A-State and PSI measures there was a significant main effect for sex of subject [A-State: $F(1,120)=4.01, p<.05$; PSI: $F(1,120)=4.06, p<.05]$. These results showed females experienced more arousal in the task situation than did males, with a mean A-State task score of 42.95 compared to 40.20 for 


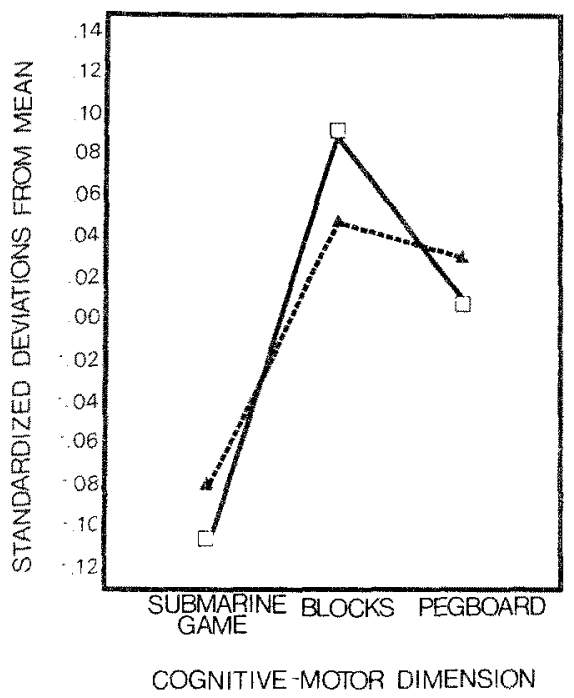

Fig. 1. A-State (D) and PSI (A) measures (standardized) as a function of task.

males, and a mean PSI task score of 16.67 vs. 13.95 for males. Identical analyses performed on each task arousal measure separately resulted in significant main effects for sex of subject, again with females having greater arousal scores than males.

The significant effects found on the PSI arousal measurement scores described above were the only significant findings found for the PSI in the experiment. Therefore, the discussion of arousal from this point on refers solely to A-State scores.

\section{Trait Anxiety}

A $4 \times 2$ repeated measures analysis of variance of the four task arousal measures, with task measure and trait anxiety level as factors, resulted in significant main effects for both factors $[F(3,444)=16.12, p<.001$ for task measure; $F(1,148)=31.44, p<.001$ for trait anxiety level]. Table I presents the mean arousal scores by task and trait anxiety level. High-traitanxious subjects had higher arousal scores for each task measure than did low-trait-anxious subjects; for both high- and low-trait-anxious subjects, the blocks test was the most arousing, followed by the pegboard and submarine game tasks in order of decreasing arousal. There were approximately equal numbers of males and females in each of the two trait anxiety levels. 
Table I. Mean A-State Scores by Task and Trait Anxiety Level ${ }^{a}$

\begin{tabular}{lcccc}
\hline $\begin{array}{c}\text { Trait anxiety } \\
\text { level }\end{array}$ & Pretask & Pegboard & Submarine & Blocks \\
\hline Low & 37.94 & 38.84 & 36.84 & 39.78 \\
High & 45.39 & 45.10 & 42.78 & 47.75 \\
\hline
\end{tabular}

a Scores could range from 20 (low) to 80 (high).

A $2 \times 3$ analysis of variance of task performance scores, examining the effect of trait anxiety and task difficulty, revealed a significant interaction $[F(2,144)=6.98, p<.002]$ on the pegboard task performance scores. Low- and high-trait-anxious subjects performed at the same level under low-difficulty conditions, but low-trait-anxious subjects performed better than high-trait-anxious subjects on the medium-difficulty task version, and vice versa in the high-difficulty condition (see Figure 2).

\section{Assessment of the Arousal-Performance Relationship}

The five audience conditions, which were designed to provide five distinct levels of arousal, did not significantly affect the subjects' level of arousal. Therefore, five post hoc arousal levels were constructed by quintiling the actual arousal scores of the subjects. Males and females were combined when scores were quintiled, even though there were sex differences in arousal. Since females were generally more aroused in the experiment than males, the arousal-performance function for males at a given difficulty level of a task would be for a lower range of arousal than the function for females. Therefore, to examine the arousal-performance relationship, we must look at the arousal level and resultant performance of subjects without regard to their sex. It should be noted, however, that the arousal-performance functions were examined for males and females separately and were found to be very similar to those for subjects as a whole.

A $5 \times 3$ (quintiled arousal level and task difficulty) analysis of variance was performed on each set of task performance scores. There was, as expected, a significant main effect for difficulty level on all three tasks: the greater the a priori difficulty level of a task version, the poorer the performance. Significant interactions between arousal condition and task difficulty level were found on both the pegboard and the submarine performance scores $[F(8,135)=2.08, p<.05$ for the pegboard; $F(8,135)=$ $3.18, p<.005$ for the submarine game], as well as a significant main effect for arousal level on the submarine game performance scores $[F(4,135)=2.76, p<.05]$. There was no significant main effect for arousal condition or significant interaction found on the blocks per- 




Fig. 2. Task difficulty by trait anxiety interaction for pegboard task performance scores. The symbol represents low-trait anxiety; $\square$, high-trait anxiety.
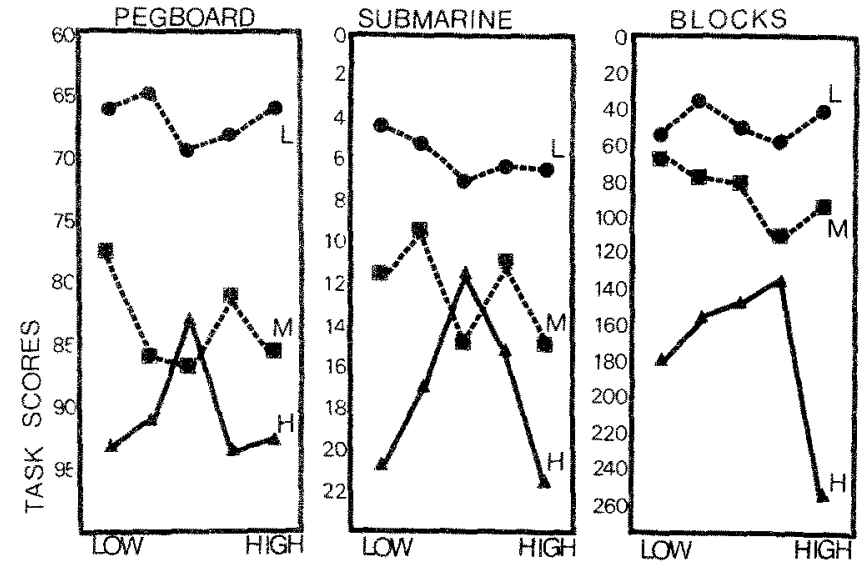

QUINTILED AROUSAL LEVEL

Fig. 3. Mean task performance scores as a function of quintiled arousal level and task difficulty $(\mathrm{L}=$ low difficulty, $\mathrm{M}=$ medium difficulty, and $H=$ high difficulty. Scores are seconds to completion for the pegboard and blocks tasks and number of guesses taken for the submarine game task.) 
formance scores. Examination of the above interactions (see Figure 3) indicated different arousal-performance functions for the three difficulty levels across the arousal conditions.

In order to test more precisely the relationship between difficulty and arousal, separate trend analyses were conducted for each difficulty level within each task. A trend analysis of the simple main effects at each difficulty level revealed significant quadratic trends for the high-difficulty level for each task, plus a quartic trend on the pegboard task [quadratic trends: $F(1,135)=5.27, p<.025$ for the pegboard; $F(1,135)=25.36, p<.001$ for the submarine game; $F(1,135)=9.24, p<.01$ for the blocks test. Quartic trend: $F(1,135)=5.98, p<.025]$. There was a significant quartic trend for the medium difficulty condition on the submarine game $[F(1,135)$ $=6.81, p<.01]$. No significant linear or nonlinear trends were found for the low-difficulty condition on any of the three tasks.

The results of the trend analyses and an examination of the arousal condition by task difficulty interactions for all three tasks appears to indicate that the inverted-U relationship between arousal and performance occurs only under high task difficulty conditions. A higher level trend (cubic or quartic) is found at the medium difficulty level, and there is no relationship under the low-difficulty condition. The pattern of the quintiled arousal condition by task difficulty interaction is nearly identical for all three tasks. The quadratic trend at the high-difficulty level was significant for all tasks, and there was no significant trend found for any task at the low-difficulty level. The pattern of the relationship at the medium-difficulty level is more complex, but seems to be accountable by either a cubic or quartic trend. This mediating effect of task difficulty thus generalizes over the cognitive-motor dimension as manipulated in this study.

\section{DISCUSSION}

The analysis of the task performance scores using the quintiled arousal conditions and task difficulty as factors gave nearly identically appearing interactions of these factors for the three tasks (see Figure 3). For all tasks, level of arousal did not affect performance under low-difficulty conditions. On the medium-difficulty task version, performance was generally better under low-arousal levels; the remainder of the relationship was complex and dependent to some degree upon the task. This may be because the medium-difficulty level is more affected by individual differences in task competence, compared to the relatively clear-cut high- and low-difficulty levels. Under high-difficulty conditions, a significant quadratic trend was found for all three tasks, with low-and high-arousal levels resulting in poor performance, and middle-range arousal levels resulting in the highest 
level of performance. We may conclude, therefore, that over the range of arousal achieved in this study, the arousal-performance function is an inverted $U$ for high-difficulty tasks, is complex and task dependent to some degree for medium-difficulty tasks, and that there is essentially no relationship for low-difficulty tasks. These functions are consistent over the cognitive-motor dimension.

The hypotheses of Fiske and Maddi (1961) and Oxendine (1970)-that the more difficult the task and the more physical energy needed for successful completion, the smaller the optimal range of arousal for that taskcannot be tested as they assume an inverted-U relationship for all difficulty levels. Since the inverted $U$ was found to occur only under high-difficulty conditions, this study casts doubt on the premises of these hypotheses, and raises the possibility that comparison of optimal arousal levels across difficulty levels cannot take place.

The patterns of the A-State and PSI means across the three tasks were very similar; the arousal level of each task relative to the others is the same for both arousal measures. The submarine game (the cognitive task) is much less arousing than either the pegboard or the blocks tasks. The pegboard task is less arousing than the blocks task, although the difference between them is much more pronounced on the A-State measure. The differentially arousing nature of these tasks may be due to differences in their evaluation potentials for the subject. Being a cognitive task, the subject's strategy or skill on the submarine game was not as visible as on the other tasks. The blocks test gave a subject's audience more areas on which to evaluate him than did the pegboard task, such as strategy and intelligence. Thus the blocks test may cause more evaluation apprehension than the pegboard task, which in turn would make subjects more worried about evaluation than the submarine game.

Females were significantly more aroused by the audience conditions (collapsed over tasks) than males, for both the A-State and PSI measures. The separate analyses for each task were consistent with this finding; the two significant main effects for sex of subject (on the submarine game A-State and the blocks test PSI) found females more highly aroused by the experimental situation.

The results of the trait anxiety analyses lend some support to those of Carron (1965) and Scanlan (1975). High-trait-anxious subjects performed better than low-trait-anxious subjects on the medium-difficulty pegboard task, and low-trait-anxious subjects performed better than high-traitanxious subjects on the high-difficulty version, replicating the findings of Carron (1965). Our results extend Carron's finding, however, as for the low-difficulty condition, both high- and low-trait-anxious subjects performed at the same level. Therefore, if the motor task is simple enough, trait anxiety level does not affect performance. The results of this study do 
not support generalizing Carron's findings to nonmotor tasks, suggesting that this effect of trait anxiety may be unique to motor tasks.

From Table I, it can be seen that high-trait-anxious subjects were uniformly more aroused over the course of the experiment than low-traitanxious subjects, as measured by the A-State scale. This replicated Scanlan's (1975) finding that state anxiety level in an experimental situation is largely a function of trait anxiety level.

To summarize, the experimenter's choice of arousal and performance measures may have a significant influence on his results. The use of a behavioral arousal measure and a high-difficulty task (of either cognitive or motor requirements) will most likely result in an inverted-U function between arousal and performance. With a medium-difficulty task, a cubic or higher order trend will probably be obtained, and with a low-difficulty task little or no relationship will be found.

The cognitive-motor requirements dimension produces different levels of arousal, which is most likely due to the different evaluative potentials of the three tasks used in the study. However, the cognitivemotor requirements dimension does not seem to affect the shape of the arousal-performance function, which is virtually the same for each difficulty level at three different places on the dimension.

Sex of subject was also a mediating factor, as females were generally more aroused by the experimental situation. The sex of subject factor is similar to the cognitive-motor factor in that it produces different levels of arousal but does not appreciably affect the shape of the arousal-performance function.

Interactions between trait anxiety level and task characteristics were only obtained for the motor task; no significant differences have been found for other task types. High-trait-anxious subjects scored higher on behavioral measures of situation-specific arousal than did low-trait-anxious subjects, but there were no differences due to trait anxiety level on the physiological arousal measure.

Therefore, the most powerful mediating factor of the arousal-performance relationship appears to be task difficulty, as three different functions were produced for the low-, medium-, and high-difficulty versions of both cognitive and motor tasks.

\section{REFERENCES}

Broadhurst, P. L. The interaction of task difficulty and motivation: The Yerkes-Dodson law revived. Acta Psychologica, 1959, 16, 321-338.

Carron, A. B. Complex motor skill performance under conditions of externally induced stress. (Unpublished master's thesis, University of Alberta, 1965.)

Cohen, J. L., \& Davis, J. H. The effects of audience status, evaluation, and time of action on performance with hidden word problems. Journal of Personality and Social Psychology, 1973, 27, 74-85. 
Cottrell, N. B. Performance in the presence of other human beings: Mere presence, audience, and affiliation effects. In E. C. Summel, R. A. Hoppe, \& B. A. Milton (Eds.), Social facilitation and imitative behavior, Boston: Allyn and Bacon, 1968.

Cottrell, N. B., Wack, D. L., Sekerak, G. J., \& Rittle, R. H. Social facilitation of dominant responses by the presence of an audience and the mere presence of others. Journal of Personality and Social Psychology, 1968, 9, 245-250.

Fiske, D. W., \& Maddi, S. R. Functions of varied experience. Homewood, Hllinois: Dorsey Press, 1961.

Green, H. J., \& Berman, I. R. A manual of selected occupational tests for use in public employment offices. University of Minnesota: Bulletins of the Employment Stabilization Research Institute, 1936, 2, pp. 5-7, 10-11, 23-31.

Hackman, J. R. Toward understanding the role of tasks in behavioral research. Acta Psychologica, $1969,31,97-128$.

Henchy, T., \& Glass, D. C. Evaluation apprehension and the social facilitation of dominant and subordinate responses. Journal of Personality and Social Psychology, 1968, 10, 446-454.

Innes, J.M., \& Young, R. F. The effect of presence of an audience, evaluation apprehension, and objective self-awareness on learning. Journal of Experimental Social Psychology, $1975,11,35-42$.

Johnston, J. E., \& Dabbs, J. M., Jr. Enumeration of active sweat glands: A simple physiological indicator of psychological changes. Nursing Research, 1967, 16, 273-276.

Katahn, M., Blanton, R. L., \& Gipson, M. T. Speed and amplitude of response as a function of anxiety and degree of conflict. Journal of Experimental Research in Personality, $1967,2,169-172$.

Martens, R. Arousal and motor performance. Exercise and Sport Sciences Review, 1974, 2, $155-188$.

Martens, R., \& Landers, D. M. Evaluation potential as a determinant of coaction effects. Journal of Experimental Social Psychology, 1972, 8, 347-359.

McLaughlin, R. J., \& Eysenck, H. J. Extraversion, neuroticism, and paired-associates learning. Journal of Experimental Research in Personality, 1967, 2, 128-132.

Oxendine, J. B. Emotional arousal and motor performance. Quest, 1970, 13, 23-32.

Paulus, P. B., \& Murdoch, P. Anticipated evaluation and audience presence in the enhancement of dominant responses. Journal of Experimental Social Psychology, 1971, 7, 280-291.

Sasfy, J., \& Okun, M. Form of evaluation and audience expertness as joint determinants of audience effects. Journal of Experimental Social Psychology, 1974, 10, $461-467$.

Scanlan, T. K. Effect of competition, trait anxiety and success-failure on the perception of threat in a competitive situation. Unpublished doctoral dissertation, University of Illinois, 1975.

Shaw, M. E. Scaling group tasks: A method for dimensional analysis. JSAS Catalog of Selected Documents in Psychology, 1973, 3, 8. (MS no. 294.)

Sorce, J., \& Fouts, G. Level of motivation in social facilitation of a simple task. Perceptual and Motor Skills, 1973, 37, 567-572.

Spielberger, C. D., Gorsuch, R. L., \& Lushene, R. E. The state-trait anxiety inventory. Palo Alto, California: Consulting Psychologists Press, 1970.

Stennett, R. G. The relationship of performance level to level of arousal. Journal of Experimental Psychology, 1957, 54, 54-61.

Wapner, S., \& Alper, T. G. The effect of an audience on behavior in a choice situation. Journal of Abnormal and Social Psychology, 1952, 47, 222-229.

Wechsler, D. Wechsler adult intelligence scale manual. New York: Psychological Corporation, 1955.

Yerkes, R. M., \& Dodson, J. D. The relation of strength of stimulus to rapidity of habit-formation. Joumal of Comparative Neurology and Psychology, 1908, 18, 459-482.

Zajone, R. B. Social facilitation. Science, 1965, 149, 269-274. 